\title{
Technè
}

La science au service de l'histoire de l'art et de la préservation des biens culturels

47 | 2019

Bernard Palissy : nouveaux regards sur la céramique française auX $\mathrm{XVI}{ }^{\mathrm{e}}$ et $\mathrm{XVII}{ }^{\mathrm{e}}$ siècles

\section{Datation par le carbone 14 de charbons de bois inclus dans un moule en plâtre du fonds Palissy}

Radiocarbon dating of charcoal inserted into a plaster mold from the Palissy's collection

Pascale Richardin

\section{OpenEdition}

Journals

Édition électronique

URL : http://journals.openedition.org/techne/1478

DOI : 10.4000/techne.1478

ISSN : 2534-5168

Éditeur

C2RMF

Édition imprimée

Date de publication : 1 juin 2019

Pagination : $42-47$

ISBN : 978-2-11-152830-7

ISSN : $1254-7867$

\section{Référence électronique}

Pascale Richardin, «Datation par le carbone 14 de charbons de bois inclus dans un moule en plâtre du fonds Palissy », Technè [En ligne], 47 | 2019, mis en ligne le 01 juin 2020, consulté le 28 juillet 2020. URL : http://journals.openedition.org/techne/1478 ; DOI : https://doi.org/10.4000/techne.1478

\section{cc) (†)}

La revue Technè. La science au service de l'histoire de l'art et de la préservation des biens culturels est mise à disposition selon les termes de la Licence Creative Commons Attribution - Pas d'Utilisation Commerciale - Pas de Modification 4.0 International. 


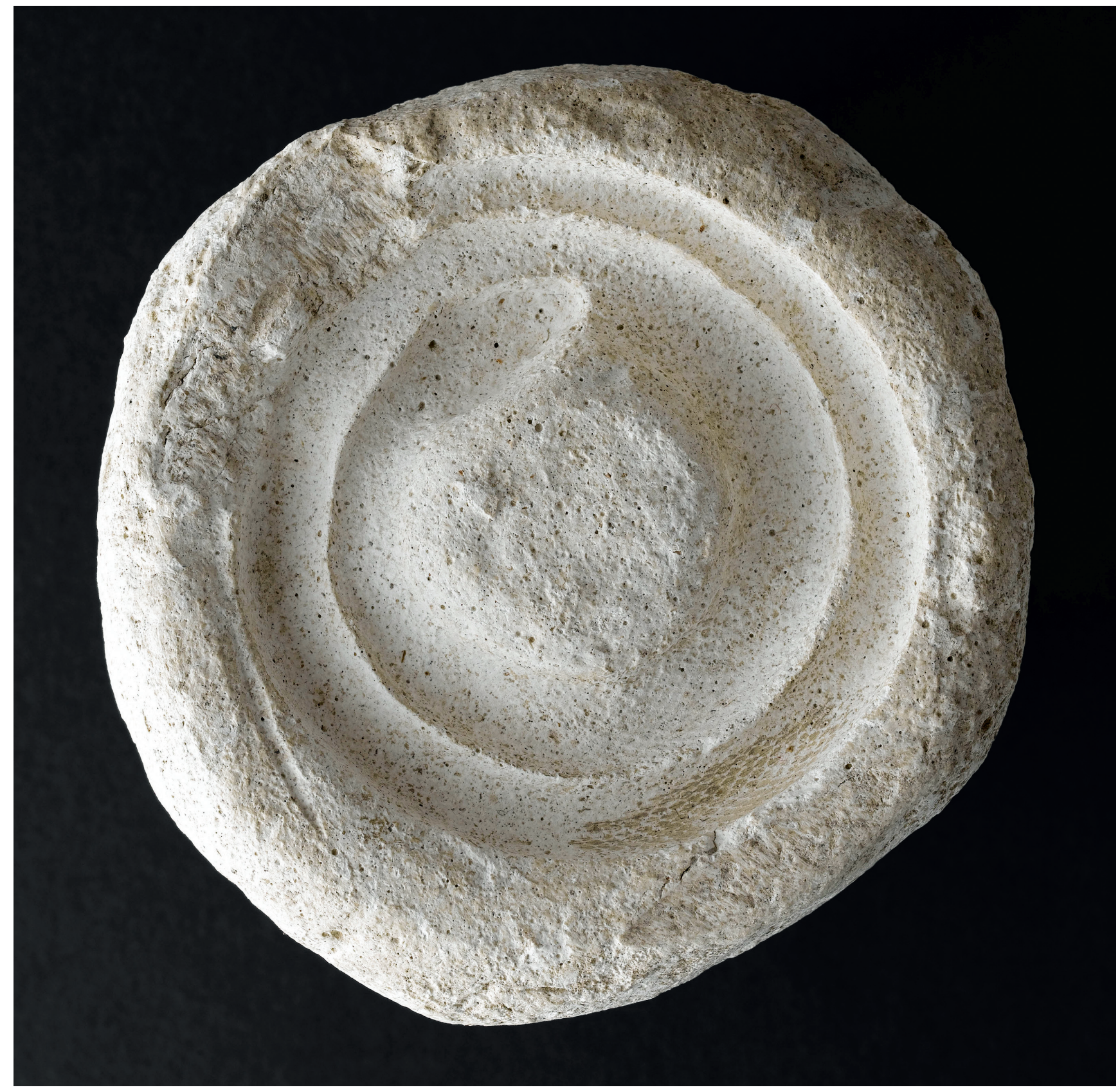

Fig. 1. Atelier de Bernard Palissy, Paris, fouilles des Tuileries, troisième quart du xvI $\mathrm{e}^{\mathrm{e}}$ siècle, Moule de serpent, plâtre (D. $11 \mathrm{~cm}$, Ép. $4 \mathrm{~cm}$ ), Écouen, musée national de la Renaissance, EP 2680. @ RMN-Grand Palais (musée national de la Renaissance, château d'Écouen)/René-Gabriel Ojéda. 
Pascale Richardin

\section{Datation par le carbone 14 de charbons de bois inclus dans un moule en plâtre du fonds Palissy}

Radiocarbon dating of charcoal inserted into a plaster mold from the Palissy's collection

Résumé. Nous avons daté par radiocarbone plusieurs petits fragments de charbon de bois, qui étaient inclus dans un moule en plâtre du fonds Palissy conservé au musée national de la Renaissance à Écouen. Le défi était double : la taille extrêmement petite des échantillons et la rareté des résultats de datation sur les charbons de bois inclus dans des plâtres. L'un des deux échantillons prélevés a permis de réaliser deux mesures. Les deux âges radiocarbone obtenus ont été combinés et la valeur calculée est : 488 \pm 22 ans BP, soit une date comprise dans l'intervalle 1410-1445 apr. J.-C.

Cette étude nous a permis de discuter de la faisabilité (préparation de l'échantillon, mesure et calibration de la date) et de la pertinence de la datation par le carbone 14 de très petits fragments de bois ou de charbon de bois, qui seraient a priori de la période Renaissance.

Mots-clés. Datation, carbone 14, charbon de bois, plâtre, Renaissance, Palissy.
Abstract. Small pieces of charcoal, inserted into a plaster mold from the Palissy's collection and kept at the national museum of Renaissance at Écouen, were submitted to radiocarbon dating. The challenge was twofold: their extremely small sample sizes and the rather rare dating results on charcoal inserted in plaster. Two measurements were done on one of the two samples taken. The radiocarbon ages were combined and the calculated value is: $488 \pm 22$ years BP, which corresponds to a calibrated date between 1410 and $1445 A D$.

This study enabled us to discuss the feasibility (sample preparation, measurement and calibration of date) and the relevance of carbon 14 dating for very small pieces of wood or charcoal, which would be a priori of the Renaissance period.

Keywords. Radiocarbon dating, charcoal, plaster, Renaissance, Palissy.

\section{Introduction}

La datation par le carbone 14 ou radiocarbone (noté ${ }^{14} \mathrm{C}$ ) est une des méthodes de datation les plus populaires ${ }^{1}$, malgré des résultats et des conclusions parfois discutés et mis en cause. D'un principe relativement simple ${ }^{2}$, la méthode demande cependant de grandes précautions lors de sa mise en œuvre, notamment lors des prélèvements (sélection, localisation et taille), du protocole de préparation des échantillons, de la calibration des mesures et de l'interprétation des résultats ${ }^{3}$.

Cette méthode de datation, qui s'applique à tous les matériaux contenant du carbone issus d'organismes ayant été vivants (végétaux ou animaux), est donc tout particulièrement adaptée à la datation du bois ou du charbon de bois ${ }^{4}$. Cependant, pour que la datation soit réalisable et significative, trois conditions sont requises : le carbone doit provenir de la période de formation de la matière (bois, graines, os...), il doit être facilement extrait ou isolé par des traitements qui n'apportent pas de carbone supplémentaire et l'échantillon doit être représentatif de l'événement à dater.

Le plâtre est un mélange pulvérulent préparé à partir de la calcination du gypse dans des fours situés relativement près des carrières. Souvent, le plâtre obtenu contient des impuretés et notamment des fragments de charbon de bois, en raison du contact avec le combustible lors de la cuisson (fig. 1). De ce fait, la datation par le carbone 14 de cette matière organique incluse dans le matériau est théoriquement possible si toutefois la quantité extraite est suffisante (entre 5 et $10 \mathrm{mg})^{5}$.

C'est pourquoi nous avons daté par radiocarbone plusieurs petits fragments de charbon de bois, qui étaient inclus dans un moule en plâtre du fonds Palissy conservé au musée national de la Renaissance à Écouen. Le défi était double : la taille extrêmement petite des échantillons ${ }^{6}$ et la rareté des résultats de datation sur les charbons de bois inclus dans des plâtres. Ce moule provient des premières fouilles du

Pascale Richardin, ingénieure de recherche, responsable du groupe Datation, département Recherche, C2RMF (pascale.richardin@culture.gouv.fr). 

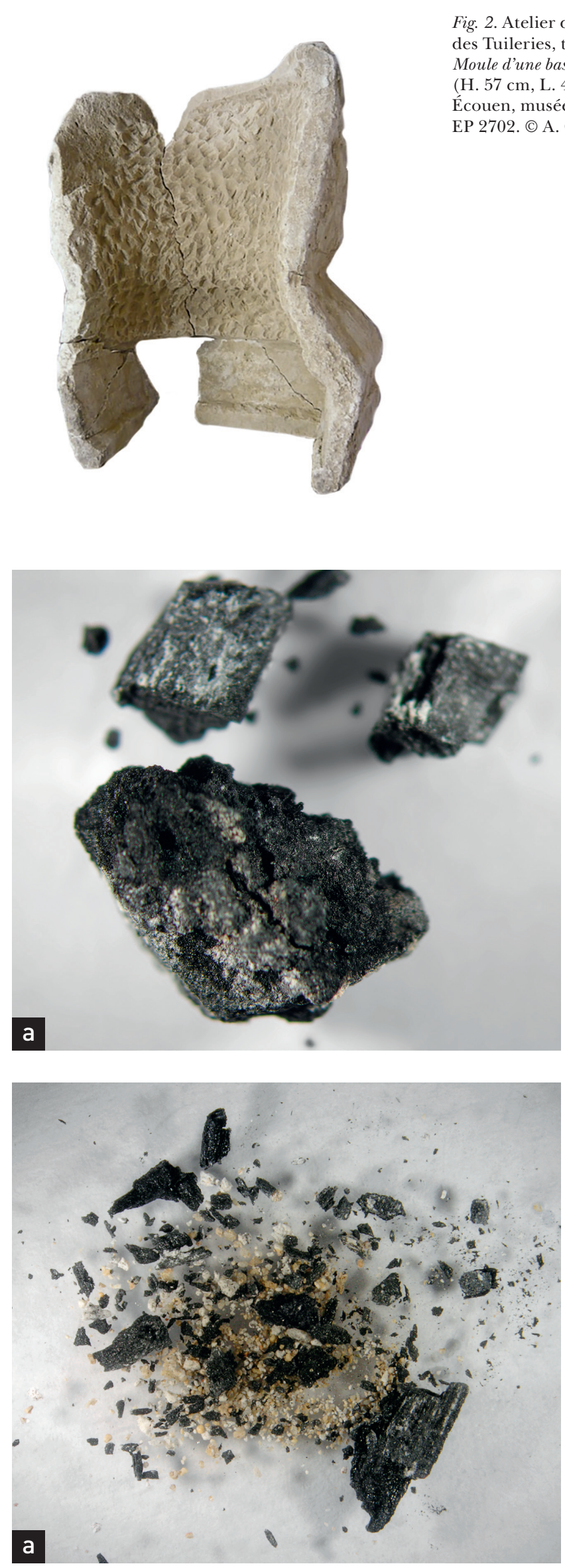

Fig. 3 b. Fragments (a et b) de l'échantillon EP2702_EF après séparation du charbon de bois et du plâtre sous la loupe binoculaire. (c) C2RMF/N. Gandolfo.
Fig. 2. Atelier de Bernard Palissy, Paris, fouilles des Tuileries, troisième quart du $\mathrm{XvI}^{\mathrm{e}}$ siècle,

Moule d'une base de terme rocaille, plâtre

(H. $57 \mathrm{~cm}$, L. $47 \mathrm{~cm}$, Pr. $27 \mathrm{~cm}$ ),

Écouen, musée national de la Renaissance, EP 2702. ๑ A. Courcelle.

Fig. 3 a. Fragments de charbon de bois (a) et du résidu de plâtre (b) de l'échantillon EP2702_EE. ( C C2RMF/N. Gandolfo.
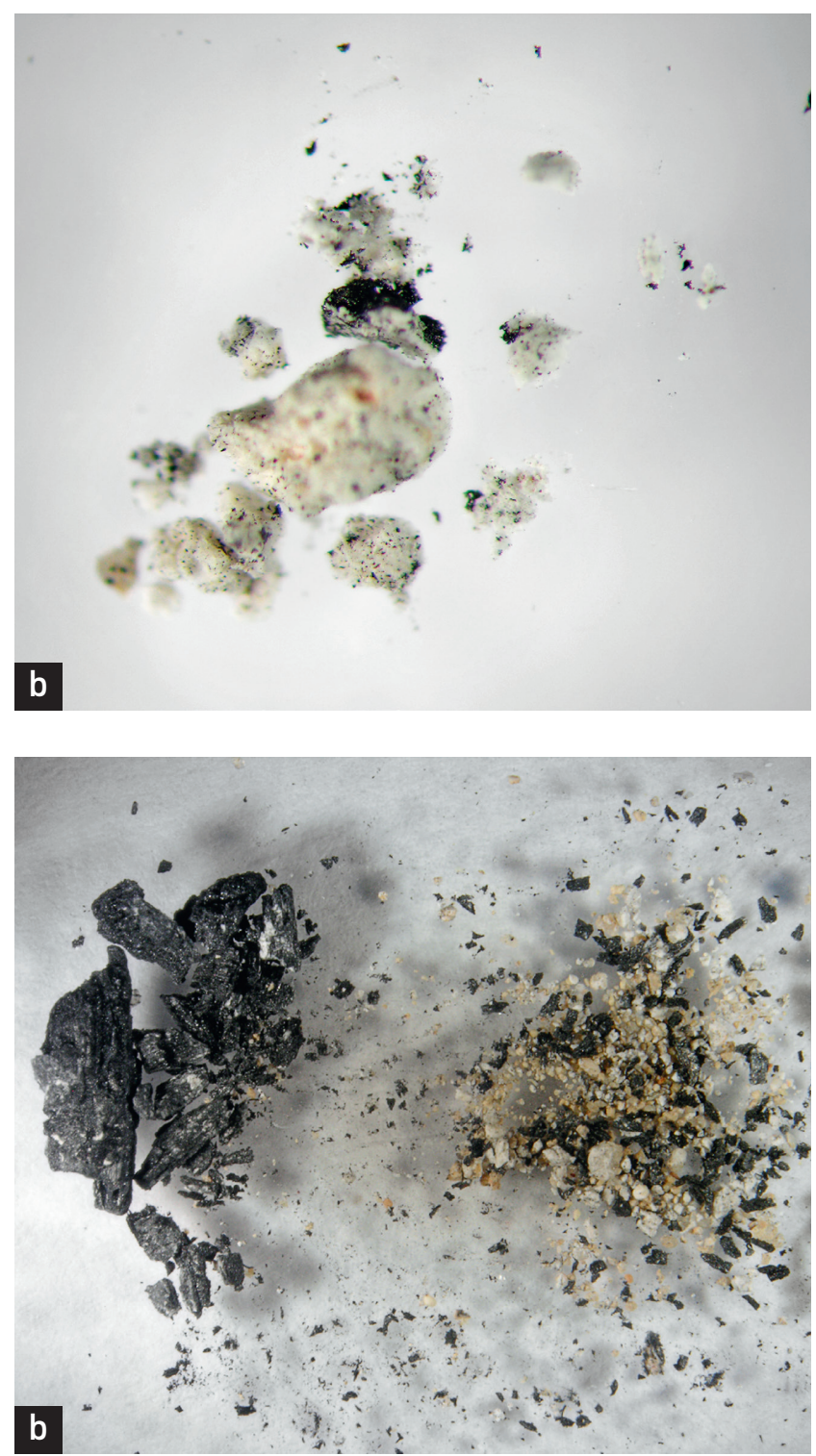
Carrousel du Louvre. Dans un contexte archéologique comme celui-ci, il ne s'agit donc pas de résoudre un problème d'authentification, mais plutôt d'évaluer la validité et de discuter de la faisabilité et de la pertinence de la datation par le carbone 14 (préparation de l'échantillon, mesure et calibration de la date) de très petits fragments de bois ou de charbon de bois, qui seraient a priori de la période Renaissance.

\section{Objets d'étude}

Le moule que nous avons étudié est un moule à creux perdu (H. $57 \mathrm{~cm}, \mathrm{~L} .47 \mathrm{~cm}, \operatorname{Pr} .27 \mathrm{~cm}$ ), qui a été restauré en $2015^{7}$. Ce moule, incomplet et cassé en plusieurs morceaux (fig. 2), est fait de deux coulées de plâtre blanc contenant de nombreux petits fragments de charbon de bois. Ce sont certains de ces fragments que nous avons prélevés et datés par le carbone 14 .

\section{Prélèvements des échantillons}

Deux échantillons ont été prélevés par Anne Bouquillon et Jacques Castaing au musée d'Écouen. L'échantillon EP2702_ EE (fig. 3 a) est composé principalement de fragments de bois de chêne et l'échantillon EP2702_EF (fig. 3 b) contient des fragments de charbon de bois de hêtre ${ }^{8}$. Ils consistent en de petits fragments (de 0,5 à $2 \mathrm{~mm}$ de long) de charbon de bois, mêlés à une quantité importante de grains de gypse. Ils ont été triés sous la loupe binoculaire à l'aide d'une lame de scalpel et des quantités d'environ $22 \mathrm{mg}$ pour EP2702_EE et $7 \mathrm{mg}$ pour EP2702_EF ont été récupérées.

\section{Préparation des échantillons et mesure du taux de carbone 14}

La préparation d'échantillons de charbon de bois suit le protocole classique (ABA) pour un échantillon d'origine végétale. Il consiste en une succession de lavages avec des solutions aqueuses d'acide chlorhydrique et de soude, et des rinçages à l'eau entre chaque lavage ${ }^{9}$. Malgré les précautions d'usage (dilution des solutions, lavage à température ambiante), la faible quantité d'échantillon récupérée après le lavage de EP2702_EF (0,16 mg, donc un rendement pondéral de $2 \%$ ) ne nous a pas permis de poursuivre la préparation chimique (combustion, graphitisation).

L'échantillon EP2702_EE nous a fourni une quantité suffisante pour préparer deux tubes pour la combustion et nous avons pu par conséquent effectuer deux mesures de ${ }^{14} \mathrm{C}$. Afin de contrôler le protocole, nous avons réalisé la préparation et la combustion des deux échantillons de charbon (Essai 1 et Essai 2) et d'un échantillon standard, qui est un charbon de bois d'Afrique du Sud (CBS) d'âge très supérieur à la limite de détection et qui sert de «blanc ». L'âge radiocarbone obtenu pour ce dernier est supérieur à 45000 ans BP. Le tableau 1 présente les âges radiocarbone obtenus pour les deux tubes d'échantillon ${ }^{10}$, accompagnés des dates calibrées obtenues avec le logiciel OxCal ${ }^{11}$ et grâce aux données de calibration les plus récentes ${ }^{12}$.

Les deux âges radiocarbone pour Essai 1 et Essai 2, représentant le même événement, peuvent être combinés et la valeur calculée par le logiciel OxCal est : 488 22 ans BP, soit une date comprise dans l'intervalle 1410-1445 apr. J.-C. (fig. 4), plage de date très restreinte (35 années).

On peut se questionner sur le niveau de confiance de la date obtenue, sachant que Bernard Palissy est né en 1510 et mort en 1590 et, de façon plus générale, sur la pertinence des datations radiocarbone mesurées pour la période de la Renaissance (1300 à 1650).

\section{Calibration de l'âge radiocarbone pour la période de la Renaissance}

Les âges ${ }^{14} \mathrm{C}$ mesurés sont transformés en dates calibrées (années réelles) grâce aux courbes de calibration et aux logiciels cités plus haut. Ainsi, à chaque âge radiocarbone (en années BP ou « avant $1950 »)$ correspond une plage de dates, dans laquelle le résultat a 95,4\% de chance de se trouver (valeur dite à 2 sigmas). Cet intervalle est plus ou moins large en fonction de la portion de la courbe de calibration considérée et de la précision de la mesure.

Pour la période de la Renaissance, la courbe de calibration présente une allure particulière. Entre les années 1500

Tableau 1. Âge radiocarbone et date calibrée pour l'échantillon EP2702_EE

\begin{tabular}{|l|l|l|l|l|}
\hline \multicolumn{2}{|l|}{ Échantillon } & $\begin{array}{l}\mathbf{N}^{\circ} \text { cible } \\
\text { (réf. UMS) }\end{array}$ & $\begin{array}{l}\text { Âge } \\
\text { radiocarbone } \\
\text { (années BP) }\end{array}$ & Date calibrée \\
\hline \multirow{3}{*}{ EP2702EE } & Essai 1 & SacA 10609 & $470 \pm 30$ & $1405 \mathrm{AD}(95,4 \%) 1460 \mathrm{AD}$ \\
\cline { 2 - 5 } & Essai 2 & SacA 10610 & $505 \pm 30$ & $\begin{array}{l}1325 \mathrm{AD}(2,6 \%) 1340 \mathrm{AD} \\
1395 \mathrm{AD}(92,8 \%) 1450 \mathrm{AD}\end{array}$ \\
\cline { 2 - 5 } & $\begin{array}{l}\text { Date } \\
\text { combinée }\end{array}$ & $/$ & $488 \pm 22$ & $1410 \mathrm{AD}(95,4 \%) 1445 \mathrm{AD}$ \\
\hline
\end{tabular}


et 1600 , la courbe présente un palier (ou «plateau») qui est donc une période «défavorable » pour l'obtention d'une date précise. Par exemple, à l'âge ${ }^{14} \mathrm{C} 300 \pm 30$ ans BP (fig. 5-1) correspond une date comprise entre 1490-1605 (avec 69,5\% de probabilité) ou entre 1610-1655 (avec $26 \%$ de probabilité), donc sur une période s'étalant sur 165 années, d'où une incertitude très large.

Si on considère l'âge ${ }^{14} \mathrm{C} 480 \pm 30$ ans BP (fig. 5-2), on remarque qu'il se trouve dans une période beaucoup plus favorable car, dans cet intervalle de temps, la pente de la courbe est la plus forte. La date calibrée obtenue est alors comprise entre 1410 et 1450 , soit sur une période de 40 années. Ainsi, l'âge obtenu pour le charbon de bois EP2702_EE est donc dans un intervalle de temps très favorable $(488 \pm 22$ ans $\mathrm{BP})$.

Autour de l'âge ${ }^{14} \mathrm{C} 580 \pm 30$ ans BP, la pente de la courbe s'inverse deux fois successivement sur une courte période (fig. 5-3). Les dates calibrées présenteront deux valeurs distinctes avec des probabilités équivalentes. Ainsi, à $580 \pm 30$ ans BP correspond une date calibrée comprise entre 13001370 (avec $65 \%$ de probabilité) et 1380-1420 (30\% de probabilité).

Enfin, pour un âge ${ }^{14} \mathrm{C}$ comme $700 \pm 30$ ans BP (fig. 5-4), la période s'étend sur deux intervalles de 40 à 50 ans espacés de 50 ans, de 1260-1310 (77\%) et 1360-1390 (18\%).

\section{La datation par le carbone 14 du bois ou du charbon de bois}

Un autre paramètre doit être pris en compte quand on date du bois ou ses dérivés : c'est le phénomène dit « effet vieux bois ».

La formation du bois se traduit par la production d'un cerne annuel à la périphérie du tronc. Un prélèvement réalisé dans le cerne externe aura une teneur en ${ }^{14} \mathrm{C}$ en équilibre avec l'atmosphère, alors que celui réalisé à la moelle de l'arbre présentera la teneur en ${ }^{14} \mathrm{C}$ de l'année de sa production. Ainsi, si la datation d'un seul cerne de croissance correspond à son année de formation, celle faite sur un fragment de bois comprenant plusieurs dizaines de cernes correspondra à une date moyenne de la production des cernes.

Lorsque les objets à dater sont issus d'arbres jeunes au moment de leur mort (moins d'une trentaine d'années), la position de l'échantillon prélevé n'aura pas d'influence sur la datation. En revanche, dans le cas d'arbres très âgés au moment de leur abattage, cette position aura une incidence : plus elle sera proche de la périphérie du tronc, plus la datation obtenue sera proche de la date d'abattage de l'arbre. À l'inverse, plus elle sera proche de la moelle, plus la datation sera éloignée de la date réelle de l'emploi du bois : on parle alors « d'effet vieux bois ${ }^{13}$ ».

Il en est de même pour le charbon de bois, puisque l'âge de l'échantillon reflétera le moment où le bois aura été coupé et non celui où il a été brûlé. Ainsi, le charbon de bois peut avoir un âge apparent qui peut être entaché d'erreurs, à



Fig. 4. Représentation graphique de la courbe de calibration avec les dates calendaires obtenues pour Essai 1 et Essai 2 (en gris) et pour la date combinée (en rouge).

(c) C2RMF/P. Richardin.

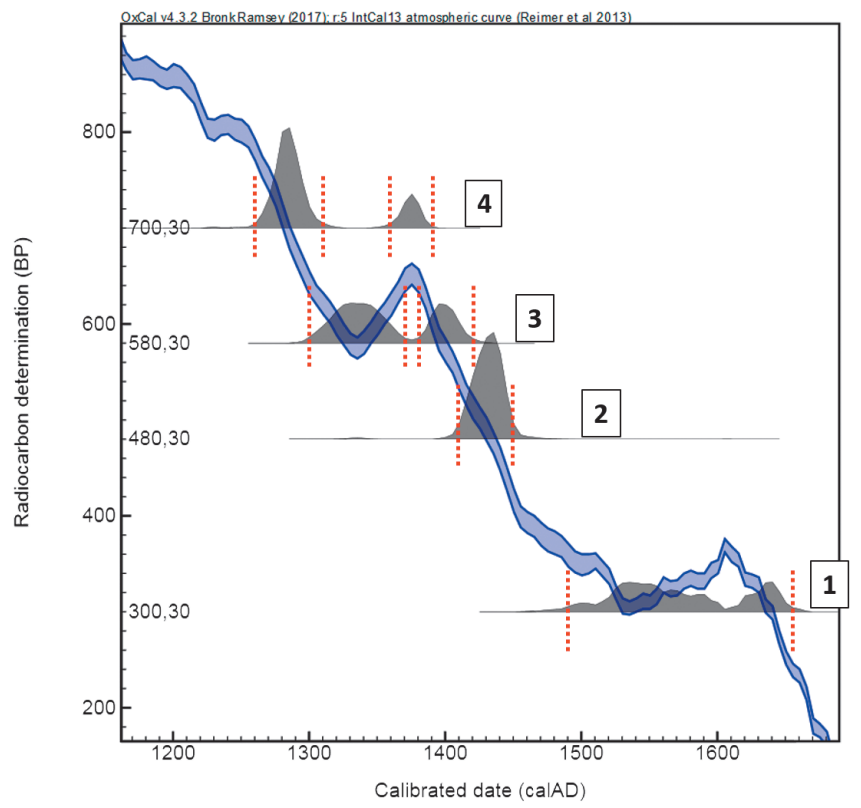

Fig. 5. Représentation graphique de la courbe de calibration avec les dates calendaires obtenues : $300 \pm 30$ ans BP (1), $480 \pm 30$ ans BP (2), $580 \pm 30$ ans BP (3) et $700 \pm 30$ ans BP (4). (c) C2RMF/P. Richardin. 
moins de provenir d'espèces à courte durée de vie ou de petites brindilles.

En conclusion, il faudra donc garder à l'esprit que cette datation fournit un terminus post quem vis-à-vis de la préparation du plâtre et n'est valable que si le matériel organique à dater est contemporain de la préparation du plâtre.

\section{Conclusion}

Sur un échantillon de charbon de bois prélevé dans un moule en plâtre de la collection Palissy du musée national de la Renaissance à Écouen, nous avons obtenu un âge radiocarbone de $488 \pm 22$ ans BP, soit une date comprise dans l'intervalle 1410-1445 apr. J.-C., intervalle de temps de faible amplitude.

Malgré une période «favorable » pour la calibration de l'âge carbone 14, la date calibrée se révèle être plus ancienne que le contexte à dater (Bernard Palissy vers 1510-1590). Cependant, il est possible que le morceau de bois utilisé dans le four ne soit pas contemporain de sa période d'utilisation. Ceci est particulièrement observé pour les bois de feuillus très résistants dans le temps et qui peuvent être utilisés après plusieurs années. Nous sommes peut-être en présence de l'effet « vieux bois », qui représente une sorte de terminus post quem ou date plancher, c'est-à-dire une date avant laquelle le bois n'a pas pu être utilisé.

\section{Remerciements}

L'auteur tient à remercier pleinement et très chaleureusement madame Anne Bouquillon (C2RMF) pour ses conseils et remarques très appropriés, ainsi que monsieur Jacques Castaing pour la réalisation des prélèvements sur l'œuvre.
Notes

1. Arnold, Libby, 1951.

2. Richardin, Gandolfo, 2013a.

3. De Reyer et al., 2016.

4. Richardin et al., Gandolfo, 2015.

5. Al-Bashaireh, Hodgins Gregory, 2011.

6. Richardin, Gandolfo, 2013b.

7. Courcelle, Picur, 2015.

8. Les identifications des essences des bois ont été réalisées par Catherine Lavier, archéodendromètre du C2RMF.

9. Richardin et al., 2015.

10. Les mesures du carbone 14 ont été réalisées par AMS (Spectrométrie de masse par accélérateur) sur l'accélérateur ARTEMIS, situé au CEA de Saclay. Les âges calendaires ont été déterminés grâce au logiciel OxCal v4.3.2 et le calcul se fait en ligne sur le site de Oxford Radiocarbon Accelerator Unit (ORAU) : https://c14.arch. ox.ac.uk/oxcal/OxCal.html. Les résultats de calibration sont considérés dans un intervalle à 2 sigmas $(2 \sigma)$, c'est-à-dire un niveau de confiance à 95,4\% et exprimés en années cal BC ou cal AD (avant ou après J.-C.).

11. Bronk Ramsey, Lee, 2013.

12. Reimer et al., 2013.

13. Stouvenot et al., 2015

\section{Bibliographie}

Al-Bashaireh K., Hodgins Gregory W. L., 2011, "AMS 14C dating of organic inclusions of plaster and mortar from different structures at Petra-Jordan", Journal of Archaeological Science, 38, p. $485-491$

Arnold J. R., Libby W. F., 1951, "Radiocarbon Dates", Science, 113(2927), p. 111-120.

Bronk Ramsey C., Lee S., 2013, "Recent and Planned Developments of the Program OxCal", Radiocarbon, 55(2-3), p. 720730.

De Reyer D., Richardin P., Nowik W., 2016, «Analyse et datation des matériaux textiles anciens », Monumental, 2, p. 104-107.

Reimer P. J. et al., 2013, "IntCal13 and Marine13 Radiocarbon Age Calibration Curves 0-50,000 Years cal BP", Radiocarbon, 55(4), p. 1869-1887.

Richardin P., Gandolfo N., 2013a, « Datation et authentification des œuvres de musée - Apport de la datation par le carbone 14 ", Spectra Analyse, 292, p. 55-60.
Richardin P., Gandolfo N., 2013b, « Datation par le carbone 14 de charbons de bois de petite taille provenant du site du Petit Paulmy », dans Marquet J.-C. et Millet-Richard L.-A. (dir.), Le Petit Paulmy, Abilly, Indre-et-Loire, Un habitat du Néolithique final de la région pressignienne, $46^{\mathrm{e}}$ supplément à la Revue archéologique du Centre de la France (RACF), p. 505-512.

Richardin P., Lavier C., Horta H., Figueroa V., Lira N., 2015, "Radiocarbon dating of Atacama (Chile) snuffs trays: an update on stylistic and chronological correlations", Radiocarbon, 57(5), p. 1-10.

Stouvenot C., Beauchêne J., Bonnissent D., Oberlin C., 2015. « Datations radiocarbone et le "problème vieux bois" dans l'arc antillais : état de la question ", dans Proceedings of the 25th International Congress for Caribbean Archeology, Porto Rico 2013, p. 459-494.

\section{Document inédit}

Courcelle A., Picur V., 2015, Étude des plâtres de la collection Palissy - Niveau $68003-n^{\circ}$ d'inv. : EP 2702. Fiche d'intervention, $3 \mathrm{p}$. 